\title{
Hydrogen diffusivity in FeAl
}

\author{
P BANERJEE and R BALASLBRAMANIAM* \\ Department of Materials and Metallurgical Engineering, Indian Institute of Technology, \\ Kanpur 208 016, India \\ MS reccived 11 February $199 ?$
}

\begin{abstract}
The room temperature diffusivity of hydrogen in a fully $\mathrm{B} 2$ ordered iron aluminide of composition $\mathrm{Fe}-358 \mathrm{~A}$ l was estimated from the experimental hydrogen depth profile to be $2.38 \times 10^{-15} \mathrm{~m}^{2} / \mathrm{s}$. The mathematical procedure utilized for data analysis has been described. The estimated diffusivity is a lower bound value due to surface trapping effects.
\end{abstract}

Keywords. Hydrogen diffusivity: iron aluminide; FeAl; mathematical analysis.

\section{Introduction}

It is well known that iron aluminides exhibit poor room temperature ductilities due to hydrogen embrittiement (Liu et al 1989). Hydrogen is produced on the surface due to the reaction of moisture with the base iron aluminide and the nascent hydrogen enters the lattice to cause embrittlement (Agarwal and Balasubramaniam 1996). One of the important factors that needs to be understood is the diffusivity of hydrogen in iron aluminides.

Zhu et al (1996) recently provided direct evidence for the production of hydrogen by reaction of moisture with iron aluminide $\mathrm{FeAl}(\mathrm{Fe}-35.8$ at \% $\mathrm{Al}$ ) surfaces. Having provided heat treatments to obtain FeAl in a fully recrystallized ordered B2 structure, they used a laser desorption mass spectrometric method to detect the presence of hydrogen after contact of iron aluminide with water or after cathodic hydrogen charging. With the aid of pulsed-laser material removal, they were also able to obtain the three dimensional distribution of trapped hydrogen atoms as a function of depth from the surface after the iron aluminide was cathodically charged with hydrogen for one day in $1 \mathrm{~N}_{2} \mathrm{SO}_{4}$. They reported the strength of the hydrogen signal (in $\mathrm{mV}$ ) that was obtained in their spectrometer as a function of depth in figure 3 of their paper. The aim of the present paper is to determine the diffusivity of hydrogen in $\mathrm{FeAl}$ using this published data.

\section{Diffusivity estimation procedure}

The experimental data of Zhu et al (1996) on hydrogen distribution as a function depth from the surface is provided in figure 1 . The data exhibits a lot of scatter especially after a depth of $20 \mu \mathrm{m}$ from the surface. The variation of $\mathrm{H}$ signal (denoted henceforth as HYs) from surface to bulk is due to the diffusing species (hydrogen) as no hydrogen signal is detected for the uncharged specimens or for specimens not exposed to water (Zhu et al 1996). Assuming $\left(C-C_{b}\right)$ to be proportional to increase in hydrogen signal over the bulk value, i.e. HYs-HYs, we obtain

$$
\frac{C-C_{b}}{C_{s}-C_{b}}=\frac{H Y s}{H Y s_{s}-H Y Y_{s}}
$$

*Author for correspondence 


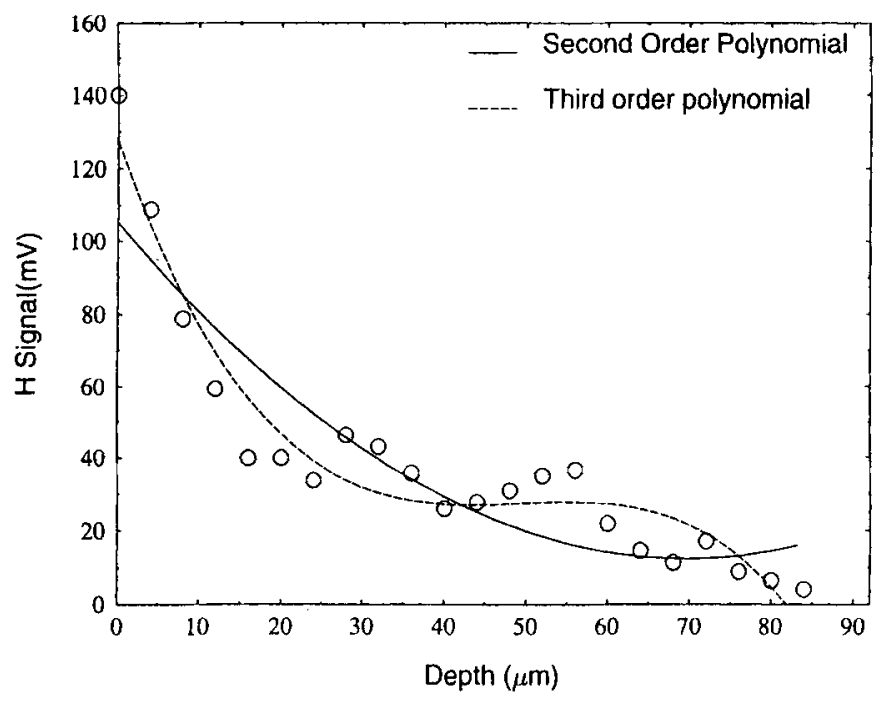

Figure 1. Data of Zhu et al (1996) showing experimental data of the strength of the hydrogen signal measured by laser desorption mass spectrometry as a function of depth in Fe-35.8 Al. The specimen was cathodically charged with hydrogen in $1 \mathrm{~N}$ sulphuric acid for 1 day prior to the experiment.

where $C_{\mathrm{s}}$ is the surface concentration of diffusing species, $C_{\mathrm{b}}$ the bulk concentration of diffusing species and $C$ the concentration of diffusing species at any point and subscripts $b$ and $s$ denote bulk and surface, respectively.

Since the specimen surfaces were flat and the depths of diffusion field were very small compared to thickness of samples $(6350 \mu \mathrm{m}$ as provided by Zhu et al (1996)), the problem may be treated as unsteady diffusion through a semi-infinite flat specimen. By assuming that the diffusion occurred through a single phase, there was no internal phase formation in the diffusion field, the diffusion coefficient was constant in the entire diffusion zone, and the surface concentration is not a function of time, (1) can be combined with the standard diffusion equation to give

$$
\frac{H Y s-H Y s_{\mathrm{b}}}{H Y s_{\mathrm{s}}-H Y s_{\mathrm{b}}}=\frac{C-C_{\mathrm{b}}}{C_{\mathrm{s}}-C_{\mathrm{b}}}=1-\operatorname{erf}\left(\frac{z}{2 \sqrt{D t}}\right)=\operatorname{erfc}\left(\frac{z}{2 \sqrt{D t}}\right)
$$

The assumptions used in the above analysis are valid. First, it is known that the composition $\mathrm{Fe}-35.8 \mathrm{Al}$ lies in the single phase region in the $\mathrm{Fe}-\mathrm{Al}$ phase diagram and therefore the assumption of diffusion taking place in a single phase field is valid. Secondly, the heat treatments provided by Zhu et al (1996) resulted in a fully ordered B2 structure and therefore the presence of a second (disordered $\alpha$ or ordered $\mathrm{DO}_{3}$ ) phase is also excluded. However, the assumption of constant surface concentration is not strictly valid because variations in the specimen potential generally occur during cathodic hydrogen charging. Therefore, $C_{\mathrm{s}}$ should be ideally eliminated from (2). $C_{\mathrm{s}}$ can be eliminated and the diffusion coefficient was determined using (2) by the following method (Roy et al 1996). A pair of points on the hydrogen signal vs depth curve was 
chosen and the following parameter $Y_{12}$ was obtained (for two given points 1 and 2) based on (2).

$$
\begin{aligned}
Y_{12} & =\frac{\left(C_{1}-C_{\mathrm{b}}\right) /\left(C_{\mathrm{s}}-C_{\mathrm{b}}\right)}{\left(C_{2}-C_{\mathrm{b}}\right) /\left(C_{\mathrm{s}}-C_{\mathrm{b}}\right)}=\frac{C_{1}-C_{\mathrm{b}}}{C_{2}-C_{\mathrm{b}}}=\frac{\operatorname{erfc}\left(z_{1} / 2 \sqrt{D t}\right)}{\operatorname{erfc}\left(z_{2} / 2 \sqrt{D t}\right)} \\
& =\frac{(\mathrm{HYs})_{1}-(\mathrm{HYs})_{\mathrm{b}}}{(\mathrm{HYs})_{2}-(\mathrm{HYs})_{\mathrm{b}}},
\end{aligned}
$$

where $(\mathrm{HYs})_{1},(\mathrm{HYs})_{2}$ and $(\mathrm{HYs})_{\mathrm{b}}$ are the values of hydrogen signals and $C_{1}, C_{2}$ and $C_{\mathrm{b}}$ are the hydrogen concentrations at locations 1,2 and in the bulk, respectively. $D$ is the only unknown parameter in (3) for a given experiment. Equation (3) can be solved by using error function table and by trial and error solution.

As mentioned earlier, there is a lot of scatter in the experimental data after a depth of about $20 \mu \mathrm{m}$. Therefore, the data was first mathematically analyzed. A quadratic fit of the data points provided a curve that could be drawn through the data points and this is shown in figure 1 . The coefficient of fit in this case was $81 \%$ which does not indicate very good fit of the experimental data. This curve was first used to estimate the hydrogen diffusivity according to the procedure outlined above. The value of the bulk hydrogen signal $\left(\mathrm{HYs}_{\mathrm{b}}\right)$ was taken as $14 \mathrm{mV}$ and the diffusivity values and average diffusivity calculated are provided in table 1 .

A better fit of the experimental data, especially in the near-surface region, was obtained by a third order polynomial fit and this is also shown in figure 1 . The correlation of fit in this case was $93 \%$. Hydrogen diffusivity was estimated by the above procedure by using $\mathrm{HYs}_{\mathrm{b}}$ from the first flat region of the curve (i.e. $28 \mathrm{mV}$ ) and the average diffusivity was found to be $1.34 \times 10^{-15} \mathrm{~m}^{2} / \mathrm{s}$ (table 1 ). The same curve was also used to determine hydrogen diffusivity by using the lowest data point (i.e. $4 \mathrm{mV}$ ) as the bulk $\mathrm{HYs}_{\mathrm{b}}$ value. This provided an average diffusivity value of $2.35 \times 10^{-15} \mathrm{~m}^{2} / \mathrm{s}$ (table 1). It is seen that similar hydrogen diffusivities are obtained for slightly different $\mathrm{HY}_{\mathrm{b}}$ used. It is again noticed that the estimated average hydrogen diffusivity values are comparable to those obtained from the quadratically fit curve. Finally, it can also be observed from the data that the first five data points (i.e. from the surface) lie on a smooth curve and hydrogen diffusivity was again estimated using just the four data points. The data for $z=0$ was not used as it is not amenable for analysis by (3). The average diffusivity estimated by this procedure was $1.41 \times 10^{-15} \mathrm{~m}^{2} / \mathrm{s}$.

The average diffusivity value calculated from the individual average diffusivities obtained by the different methods of analysis is $2.38 \times 10^{-15} \mathrm{~m}^{2} / \mathrm{s}$. This could be taken

Table 1. Diffusivity values and average diffusivity of hydrogen in $\mathrm{Fe}-35.8 \% \mathrm{Al}$ estimated in the present study.

\begin{tabular}{lccc}
\hline Analysis method & $\begin{array}{c}\text { Bulk HYs } \\
(\mathrm{mV})\end{array}$ & $\begin{array}{c}D_{\mathrm{H}} \\
\left(\mathrm{m}^{2} / \mathrm{s} \times 10^{15}\right)\end{array}$ & $\begin{array}{c}D_{\mathrm{H}, \text { av }} \\
\left(\mathrm{m}^{2} / \mathrm{s} \times 10^{15}\right)\end{array}$ \\
\hline Quadratic fit & 14 & $5 \cdot 84,3.86,3.83,4 \cdot 2$ & 4.43 \\
Third order & 28 & $1 \cdot 3,1 \cdot 6,1 \cdot 01,1.43$ & 1.34 \\
$\quad$ Polynomial & 4 & $3.04,2.2,1 \cdot 18,2.96$ & 2.35 \\
Actual data & 4 & $1 \cdot 28,1 \cdot 63,1.31,1.43$ & 1.41 \\
& & Average & 2.38 \\
\end{tabular}


as the diffusivity of hydrogen in $\mathrm{Fe}-35.8 \mathrm{Al}$ determined in the present analysis, because averaging a set of values is expected to diminish the estimation errors further. This is the first reported value of hydrogen diffusivity in $\mathrm{Fe}-35.8 \mathrm{Al}$.

\section{Comparison with Literature}

The diffusivity of hydrogen has been reported recently in iron aluminide of composition (in at \%) Fe-28 Al-5.0 Cr-0.5 Nb-0.5 Mo-0.2 C-0.2 B-0.1 $\mathrm{Zr}$ (Chiu et al 1996). In a study on the environment sensitive cracking of this aluminide, they determined the room temperature diffusivity of hydrogen in the intermetallic by the time-lag technique (Devanathan and Stachurski 1962) to be $1.6 \times 10^{-9} \mathrm{~m}^{2} / \mathrm{sec}$. They concluded that the reported value of hydrogen diffusivity was reasonable as it was of the same order of magnitude as that for hydrogen diffusivity in BCC metals like $\alpha-\mathrm{Fe}, \mathrm{V}$ and $\mathrm{Nb}$ (Völkl and Alefeld 1978). The estimated hydrogen diffusivity in FeAl is lower than that for the $\mathrm{Fe}_{3} \mathrm{Al}$-based aluminide.

Limited data is available in the literature on the room temperature hydrogen diffusivity for $\mathrm{Al}$ and $\mathrm{Al}$ alloys and these have been summarized by Thakur and Balasubramaniam (1996). The estimated hydrogen diffusivity in FeAl is lower than that for hydrogen diffusion in aluminum. The hydrogen diffusivity in pure iron has been determined by several authors and the available data have been critically reviewed (Völkl and Alefeld 1975, 1978; Kiuchi and McLellan 1983). There is a large degree of discrepancy in the data obtained for well-annealed b.c.c. iron and the degree of discrepancy increases sharply with decreasing temperature (Kiuchi and McLellan 1983). The measured diffusivities of several investigators begin a sharp drop around $300 \mathrm{~K}$. The reasons proposed to explain these discrepancies are the presence of intrinsic trapping sites in the structure of well annealed iron, differing states of surface oxidation and the presence of non-intrinsic defect sites in the bulk of the material (like impurity atoms, grain boundaries and dislocations) (Kiuchi and McLellan 1983). Kiuchi and McLellan (1983) also analyzed the available data on hydrogen diffusivity in wellannealed iron and concluded that the techniques that used electropolished and $\mathrm{Pd}$-coated surfaces in the gas equilibration and electrochemical methods provided reliable diffusivities. The hydrogen diffusivity calculated from these reliable data at $300 \mathrm{~K}$ is about $10^{-8} \mathrm{~m}^{2} / \mathrm{s}$. This is higher than that calculated above for FeAl in the present study. One reason for the lower diffusivity measured could be the presence of a large atomic percent of $\mathrm{Al}$ which would act as blocking sites for hydrogen diffusion result in a lower diffusivity value. Secondly, it has been reported that data obtained from specimens containing bulk or surface trapping sites produce lower diffusivity values (Kiuchi and McLellan 1983). In the case of the experiments of Zhu et al (1996), the specimens were not coated with $\mathrm{Pd}$ before hydrogen charging and therefore the absence of surface trapping sites cannot be precluded. The low hydrogen diffusivity estimated in FeAl could be due to surface and bulk trapping effects and this may be taken as a lower bound value.

\section{Conclusions}

The diffusivity of hydrogen in $\mathrm{Fe}-35.8 \mathrm{Al}$ was estimated by mathematical analysis of hydrogen concentration versus depth data of Zhu et al (1996) by applying the standard 
diffusion equation. It was found to be $2.38 \times 10^{-15} \mathrm{~m}^{2} / \mathrm{s}$ at room temperature. The estimated hydrogen diffusivity is a lower bound value due to surface trapping effects.

\section{References}

Agarwal A and Balasubramaniam R 1996 Bull. Mater. Sci. 1991

Chiu H, Qiao L and Mao X 1996 Scr. Mater. 34963

Devanathan M A V and Stachurski Z O 1962 Proc. R. Soc. A270 90

Kiuchi K and McLellan R B 1983 Acta Metall. 31961

Liu C T, Lee E H and McKamey C G 1989 Scr. Metall. Mater. 23875

Roy T K, Balasubramaniam R and Ghosh A 1996 Scr. Mater. 341425

Thakur C and Balasubramaniam R 1996 J. Mater. Sci. Lett. 151397

Völk1 J and Alefeld G 1975 in Diffusion in solids (eds) A S Nowick and J J Burton (New York: Academic Press) p. 231

Völkl J and Alefeld G (eds) 1978 in Hydroyen in metals I (Berlin: Springer Verlag) p. 321

Zhu Y F, Liu C T and Chen C H 1996 Scr. Mater. 351435 